\title{
Metformin Improves Glycemic Variability in Adults with Type 1 Diabetes Mellitus: an open-label randomized control trial
}

\author{
XIUZHEN zhang ${ }^{1}$, Dan $\mathrm{Xu}^{1}$, ping $\mathrm{xu}^{1}$, Shufen Yang${ }^{1}$, Qingmei Zhang ${ }^{1}$, Yan $\mathrm{Wu}^{1}$, and \\ Fengyi Yuan ${ }^{1}$ \\ ${ }^{1}$ Affiliation not available
}

January 2, 2021

\begin{abstract}
Introduction: Metformin has been demonstrated to enhance cardioprotective benefits in type 1 diabetes(T1DM). Although glycemic variability $(\mathrm{GV})$ is associated with increased risk of CVD in diabetes, there is a scarcity of research evaluating the effect of metformin on GV in T1DM. Objectives: In the present study, the effects of adjuvant metformin therapy on GV and metabolic control in T1DM were explored. Patients and methods: A total of 65 adults with T1DM were enrolled and subjected to physical examination, fasting laboratory tests and continuous glucose monitoring, and subsequently randomized 1:1 to 3 months of 1000- $2000 \mathrm{mg}$ metformin daily add-on insulin (MET+INS, n=34) or insulin (INS, n=31). After, baseline measurements were repeated. Results: The mean amplitude of glycemic excursions was substantially reduced in the MET+INS group, compared with the INS group $(-1.47 \pm 3.39 \mathrm{mmol} / \mathrm{L}$ versus $1.05 \pm 4.24 \mathrm{mmol} / \mathrm{L}, \mathrm{P}=0.012)$. In parallel, the largest amplitude of glycemic excursions $(-2.28 \pm 4.71 \mathrm{mmol} / \mathrm{L}$ versus $1.77 \pm 5.71 \mathrm{mmol} / \mathrm{L}, \mathrm{P}=0.003)$, the standard deviation of blood glucose $(-$ $0.62 \pm 1.15 \mathrm{mmol} / \mathrm{L}$ versus $0.08 \pm 1.23 \mathrm{mmol} / \mathrm{L}, \mathrm{P}=0.023)$, and the coefficient of variation $(-6.08 \pm 12.31 \%$ versus $2.29 \pm 11.57 \%$, $\mathrm{P}=0.008)$ all demonstrated improvement in the MET + INS group, compared with the INS group. Significant reduction in the insulin dose, body mass index and body weight were observed in patients with MET+INS, not those with INS. Conclusion: Additional metformin therapy improved GV in adults with T1DM, as well as improving body composition and reducing insulin requirement. Hence, metformin as adjunctive therapy has potential prospects in reducing the CVD risk in patients with T1DM in the long term.
\end{abstract}

Metformin Improves Glycemic Variability in Adults with Type 1 Diabetes Mellitus: an open-label randomized control trial

Short title: Metformin improves glycemic variability in type 1 diabetes

Xiuzhen Zhang, Dan Xu, Ping Xu, Shufen Yang, Qingmei Zhang, Yan Wu, Fengyi Yuan

Department of Endocrinology and Metabolism, Shenzhen People's hospital ,The Second Clinical Medical College,Jinan University,1017 Dongmen North Road, Shenzhen, Guangdong, China

518020

Corresponding author: Fengyi Yuan, M.D. Department of Endocrinology and Metabolism, Shenzhen People's hospital ,The Second Clinical Medical College,Jinan University,1017 Dongmen North Road, Shenzhen, Guangdong, China, 518020, Tel:86-25533018, Email:janexz521@outlook.com

Conflict of interest: none declared.

Acknowledgements

We thank all of the patients participated in this study. We also thank the colleagues that devoted their efforts to this study, including dietician, diabetic educator, nurses, and technicians. 
What' new?

Glycemic variability $(\mathrm{GV})$ is increasingly becoming recognized as a contributing risk factor of cardiovascular disease in type 1 diabetes mellitus (T1DM). And GV metrics in patients with T1DM with intensive insulin therapy is far from satisfactory. We found that 3 months of metformin therapy improved GV in patients with T1DM when compared with control participants. In addition, T1DM patients with a higher BMI may benefit more in aspects of GV from additional metformin therapy than those with lower BMI.

Metformin Improves Glycemic Variability in Adults with Type 1 Diabetes Mellitus: an open-label randomized control trial

Abstract

Introduction: Metformin has been demonstrated to enhance cardioprotective benefits in type 1 diabetes(T1DM). Although glycemic variability (GV) is associated with increased risk of CVD in diabetes, there is a scarcity of research evaluating the effect of metformin on GV in T1DM.

Objectives: In the present study, the effects of adjuvant metformin therapy on GV and metabolic control in T1DM were explored.

Patients and methods: A total of 65 adults with T1DM were enrolled and subjected to physical examination, fasting laboratory tests and continuous glucose monitoring, and subsequently randomized 1:1 to 3 months of 1000- $2000 \mathrm{mg}$ metformin daily add-on insulin (MET+INS, n=34) or insulin (INS, n=31). After, baseline measurements were repeated.

Results: The mean amplitude of glycemic excursions was substantially reduced in the MET+INS group, compared with the INS group $(-1.47 \pm 3.39 \mathrm{mmol} / \mathrm{L}$ versus $1.05 \pm 4.24 \mathrm{mmol} / \mathrm{L}, P=0.012)$. In parallel, the largest amplitude of glycemic excursions $(-2.28 \pm 4.71 \mathrm{mmol} / \mathrm{L}$ versus $1.77 \pm 5.71 \mathrm{mmol} / \mathrm{L}, P=0.003)$, the standard deviation of blood glucose $(-0.62 \pm 1.15 \mathrm{mmol} / \mathrm{L}$ versus $0.08 \pm 1.23 \mathrm{mmol} / \mathrm{L}, P=0.023)$, and the coefficient of variation $(-6.08 \pm 12.31 \%$ versus $2.29 \pm 11.57 \%, P=0.008)$ all demonstrated improvement in the MET+INS group, compared with the INS group. Significant reduction in the insulin dose, body mass index and body weight were observed in patients with MET+INS, not those with INS.

Conclusion: Additional metformin therapy improved GV in adults with T1DM, as well as improving body composition and reducing insulin requirement. Hence, metformin as adjunctive therapy has potential prospects in reducing the CVD risk in patients with T1DM in the long term.

Keywords :Continuous Glucose Monitoring; Glycemic variability; Metformin; Type 1 diabetes mellitus;

Introduction

Amongst the background of the rising incidence of Type 1 diabetes mellitus (T1DM) across the globe, public concern has reached a new high [1]. Despite progress in diabetes care, as the leading cause of mortality in T1DM[2], cardiovascular disease (CVD), contributes to a two- to four-times higher death rate in patients with T1DM, compared with the general population [3, 4]. Diabetes Control and Complications Trials (DCCT) have exhibited that intensive insulin therapy attenuated atherosclerosis in T1DM patients, which was primarily ascribed to the reduction in hemoglobin A1c (HbA1c) level [5]. As the standard metric of glycemic control, the HbA1c level is ideally maintained as close to normal as possible for the purpose of reducing the incidence rate of long-term diabetic complications [6]. However, the titration of increased insulin dosage is usually accompanied with weight gain and glycemic variability (GV). Further, with the increasing prevalence of obesity and overweight in patients with T1DM $[7,8]$, insulin resistance (IR) considered to increase the CVD risk has become prominent in these individuals $[9,10]$. Moreover, a culmination of evidence proposes that GV is integral in predicting adverse clinical outcomes in patients with diabetes, including hypoglycemia, diabetic complications as well as mortality[11], emerging as one of the core treatment targets of potential therapeutic strategies. High GV is an independent risk factor of CVD and could lead to further complications than constant hyperglycemia [12]. For this reason, novel approaches to flattening glucose fluctuations that will reduce the risk of CVD in T1DM are in dire need. 
For over five decades, metformin is an oral antihyperglycemic drug that has been used extensively in the treatment of type 2 diabetes (T2DM). Prior research has reported that metformin achieved glycemic control by lowering hepatic glucose output, increasing the glucose uptake in muscle, and decreasing the intestinal carbohydrates absorption rate, as well as improving insulin sensitivity $[13,14]$. The UK Prospective Diabetes Study (UKPDS) revealed that metformin reduced the CVD risk in patients with T2DM[15]. Metformin is not recommended by most guidelines, and the role thereof in adjuvant therapy for T1DM has drawn increasing concern in recent years. Aside from reduction in the daily insulin dose and body weight, direct improvements in insulin sensitivity and carotid intima-media thickness (cIMT) were also detected in T1DM patients after additional metformin therapy, indicating that this therapeutic strategy can form potential CVD risk protection [16-18].

On the basis of present knowledge, whether metformin as adjuvant therapy could help reduce GV in patients with T1DM has not yet been illustrated. Therefore, the present 3-month randomized-control trial was conducted in accordance with the following objectives: to evaluate the effect of additional metformin in insulin treatment on the primary outcome of GV and HbA1c, in addition to other parameters of glycemic control and anthropometric indexes.

Methods and Patients

The present trial was a 3-month open-label, randomized, controlled clinical trial. Subjects with T1DM were consecutively recruited between July 2017 and July 2019 at the outpatient clinic of the Department of Endocrinology and Metabolism of the Shenzhen People's hospital (The Second Clinical Medical College,Jinan University). This trial was registered at clinicaltrials.gov (NCT03590262) and conducted in accordance with the Declaration of Helsinki and the International Conference of Harmonization-Good Clinical Practice. The study protocol was approved by the Ethics Committee of the hospital and written informed consent was obtained from each participant. The inclusion criteria included: age range of 18 to 75 years, T1DM (positive for [?]1 diabetes mellitus-associated autoantibody), and treated with continuous subcutaneous insulin infusion or multiple daily injections at a stable regimen, with self-monitoring of blood glucose (SMBG) at least three times per day for at least 1 month being required prior to participation in the study. The exclusion criteria consisted of: resting blood pressure (BP) $>140 / 90 \mathrm{~mm} \mathrm{Hg}$, smoking, medications affecting insulin sensitivity (steroids, immunosuppressants, noninsulin antidiabetic agents), history of CVD, hypertension, renal failure defined as glomerular filtration rate $<45 \mathrm{~mL} / \mathrm{min} / 1.73 \mathrm{~m} 2$, diabetic ketoacidosis or severe illness within 30 days, inability to tolerate [?]500 mg metformin twice per day and pregnancy. The same diet and physical activity instructions were given by the same dietician and diabetic educator to all patients before randomization, and compliance was reinforced at each follow-up.

Study Design

After preliminary screening, 65 eligible participants were recruited and randomized by a ratio of 1:1 into a group receiving 1000- $2000 \mathrm{mg}$ metformin daily add-on insulin therapy (MET+INS, n=34) or a group receiving insulin treatment only (INS, $\mathrm{n}=31$ ), with the trail lasting 3 months. The mean metformin dose was $1500 \mathrm{mg} / \mathrm{d}$ (range, 1000-2000 mg/d), which was adjusted pursuant to the patient's drug tolerance, while the insulin dosage was adjusted predicated on SMBG. All participants were subjected to a comprehensive evaluation at baseline and a 3-month follow-up visit, including physical examination (BP, weight, height), data concerning lifestyle (physical activity and eating habit), frequency of hypoglycemia and the daily insulin regimen.

\section{Laboratory Measurements}

Blood analyses that included serum glucose, total cholesterol (TC), triglyceride (TG), high- density lipoprotein- cholesterol (HDL-C), low-density lipoprotein- cholesterol (LDL-C) were performed at the central laboratory of the hospital by using a biochemical analyzer (Modular Analytics, Roche, Mannheim, Germany).These parameters were investigated in all participants at fasting during the first visit, and subsequently repeated after completion of the 3-month follow-up. 


\section{CGM Measurements and Parameter Calculation}

Glucose levels were continuously monitored in all participants by professional retrospective CGM (iPro2, Medtronic Minimed Inc., Northridge, CA, USA) for 72 hours at baseline and at the end of the 3-month intervention. All participants were required to do pre-prandial SMBG four times a day (before breakfast, lunch, dinner and before bedtime) with a glucometer (Accu-Chek Mobile, Roche Diagnostics, Mannheim, Germany) for calibrating the CGM. The accuracy of the glucometer was calibrated by fasting blood glucose levels tested in the central laboratory, and the deviation was less than $15 \%$. Consecutive 72 -hour calibrated glucose profiles of each participant at baseline and after the 3-month intervention were recorded for further statistical analysis. CGM data from iPro2 sensor was downloaded via Carelink iPro for analysis.

Parameters of GV were calculated, such as the standard deviation of blood glucose (SDBG), mean amplitude of glucose excursions (MAGE), largest amplitude of glycemic excursions (LAGE), coefficient of variation (CV), and mean of daily differences (MODD). The time in range (TIR, glucose range of 3.9-10.0 mmol/L during a 24-hour period) and mean sensor glucose (MSG) were also calculated from the CGM data.

\section{Outcomes}

The primary outcomes were changes in MAGE and HbA1c from baseline to 3 months, while the secondary outcomes were changes in SD, LAGE, CV, MODD, TIR, BMI, BP, lipid profiles and the daily insulin dose at the 3-month follow-up, compared with baseline. Safety data includes the incidence of all symptomatic or biochemically proven hypoglycemic episodes $(<2.8 \mathrm{mmol} / \mathrm{L})$ and medication adverse events.

Power Calculations and Statistical Analyses

Owing to being a pilot study, the sample size was estimated pursuant to the feasibility of conducting the study, with a convenience sample size of 70 ultimately being adopted. Descriptive statistics were employed to summarize baseline characteristics as mean +- SD for normally distributed data and as median (interquartile range $[\mathrm{IQR}]$ ) for non-normally distributed data. Variables were checked for the distributional assumption of normality with normal plots, in addition to Kolmogorov-Smirnov and Shapiro-Wilks tests, and categorical variables were expressed as percentages. In the univariate comparisons between the MET+INS group and the INS group, categorical variables were compared by chi-squared tests, while continuous variables were compared by t-tests or Wilcoxon-Mann-Whitney tests as appropriate. A p-value of $<0.05$ (two-tailed) was considered statistically significant. All statistical analyses were conducted by utilizing the Statistical Package for the Social Sciences (version 23.0; SPSS Inc., Chicago, IL, USA).

Results

A total of 70 patients participated, with 5 of them being ineligible or lost to follow up. Among the 65 eligible randomized participants, 3 participants ( 2 in the MET+INS group, and 1 in the INS group) did not complete the study protocol. Figure 1 exhibits the diagram depicting the randomization and follow-up visit sample sizes, while baseline clinical characteristics of the 65 patients with T1DM are summarized in Table 1. The mean age of participants was $31+-10$ years old, and the median duration of diabetes was 8 years (interquartile range, 2-15years). The mean daily insulin dose was $0.63+-0.09 \mathrm{U} / \mathrm{kg}$ of body weight and BMI was $23.0+-1.8 \mathrm{~kg} / \mathrm{m} 2$. The baseline characteristics of patients with T1DM randomized into two groups that finished the study are presented in Table 2. There were no major differences in sex, age, duration of diabetes, BMI, or parameters of metabolic control (HbA1c value, lipid profiles) between the two groups at baseline. Further, no substantial differences in the CGM parameters, such as MAGE, LAGE, SDBG, MODD, CV, TIR or MSG were found between the two groups at baseline.

Effect of Metformin on Glycemic End Points

The results of the 3-month treatments with MET+INS and INS for the investigated variables are revealed in Table 3. The primary outcome of MAGE demonstrated significant improvement in the MET+INS group, as against the INS group $(-1.47+-3.39 \mathrm{mmol} / \mathrm{L}$ versus $1.05+-4.24 \mathrm{mmol} / \mathrm{L}, P=0.012$, Figure $2 \mathrm{~A})$. Additionally, SDBG (-0.62+-1.15 mmol/L versus 0.08+-1.23 mmol/L , $P=0.023$, Figure 2B), LAGE(-2.28+-4.71 mmol/L 
versus $1.77+-5.71 \mathrm{mmol} / \mathrm{L}, P=0.003$, Figure $2 \mathrm{C})$, and $\mathrm{CV}(-6.08+-12.31 \%$ versus $2.29+-11.57 \%, \mathrm{p}=0.008$, Figure 2D) all exhibited improvement in the MET+INS group, compared with the INS group. MSG was reduced $(-0.13+-0.58 \mathrm{mmol} / \mathrm{l}$ versus $0.23+-0.75 \mathrm{mmol} / \mathrm{l}, P=0.038)$ with additional metformin. Reduction in HbA1c $(-0.05+-0.22 \%$ versus $0.03+-0.28 \%, P=0.217)$ was discovered in the MET+INS group compared with the INS group, but did not reach statistical significance. In parallel, changes in TIR displayed no major difference between the two groups.

\section{Subgroup Analysis of Glycemic Variability and Glycemic Control in the MET+INS group}

The patients in the MET+INS group were further divided into two subgroups on the basis of the median of baseline BMI $(23.2 \mathrm{~kg} / \mathrm{m} 2)$, FPG $(8.6 \mathrm{mmol} / \mathrm{L})$ and HbA1c $(8.2 \%)$, respectively (Table 4$)$. The patients with a higher BMI presented greater reduction in MAGE $(-2.76+-3.12 \mathrm{mmol} / \mathrm{L}$ versus $0.19+-3.08 \mathrm{mmol} / \mathrm{L}$, $P=0.012)$ and CV $(-9.96+-11.49 \%$ versus $-1.10+-11.88 \%, P=0.041)$ as against those with a lower BMI. Yet, the changes in SDBG, LAGE, MODD, TIR, MSG and HbA1c were not especially different between the two subgroups. No significant difference in the changes of GV and glycemic control was found between patients with different levels of FPG or HbA1c at baseline.

Effect of Metformin on Insulin Requirement and Markers of Body Composition

Significant reduction in daily insulin dose per body weight $(-0.02+-0.01 \mathrm{U} / \mathrm{kg}$ of body weight versus $0.00+-$ $0.02 \mathrm{U} / \mathrm{kg}$ of body weight, $P$ ¡0.001; Figure $3 \mathrm{~A}$ and Table 5$)$ was identified in the MET+INS group as against the INS group. Both body weight $(-0.4+-0.6 \mathrm{~kg}$ versus $0.2+-0.5 \mathrm{~kg}, P i 0.001$; Figure $3 \mathrm{~B}$ and Table 5$)$ and BMI (-0.2+-0.2 kg/m2 versus $0.1+-0.2 \mathrm{~kg} / \mathrm{m} 2, P$ ¡0.001; Figure $3 \mathrm{C}$ and Table 5$)$ were substantially reduced in the MET+INS group compared with the INS group after the 3-month intervention.

\section{Effect of Metformin on Other Traditional CVD Risk Factors}

The adjuvant metformin therapy resulted in no notable change of lipids profile (TG, TC, HDL-c and LDL-c), systolic BP and diastolic BP between the two groups (Table 5).

\section{Safety Data}

Among the 65 randomized participants, only 3 participants (4.6\%) failed to complete the study protocol. There were no severe adverse events, with only minor gastrointestinal side effects (nausea, flatulence, reduced appetite, diarrhea) being reported in 8 participants in the MET+INS group (23.5\%). This led to de-escalation in the metformin dose in 4 participants $(11.8 \%)$, while for the other 4 patients, these symptoms spontaneously resolved within 7 days. Hence, all participants tolerated at least $1000 \mathrm{mg}$ metformin per day. Hypoglycemia events were recorded in 4 subjects with MET+INS and 3 subjects with INS, and no sever hypoglycemia events were identified during the whole study. No major changes in serum creatinine, alanine aminotransferase, aspartate aminotransferase were observed between the two groups (data not shown).

\section{Discussion}

In the present randomized, open-label, controlled trial, a slight decline in the HbA1c level in the MET+INS group was observed, in contrast to the rising trend in the INS group. However, there was no significant difference of these changes between the two groups.

In recent years, a profusion of attempts have been made to assist T1DM patients in achieving near-normal glycemic control, including advancement in diabetes management through the development of insulin analogs, insulin infusion devices, as well as glucose monitoring systems. Despite these attempts, the current situation is far from satisfactory [19-21]. Previous clinical studies and meta-analyses have reported that metformin, as an adjuvant therapy to T1DM, has minimal benefits for glycemic control [7, 16, 18, 22, 23]. Conversely, new evidence has revealed modest improvements in the HbA1c level of T1DM patients with adjuvant metformin therapy. One meta-analysis highlighted that metformin reduced the HbA1c level by $0.26 \%$ in T1DM [24]. These discrepancies may be attributed to different study durations with a 3-month cutoff point, implying that the reduction in the HbA1c level caused by metformin could not sustain over time[24]. Moreover, a 
study of T1DM patients under real-world conditions reported that the HbA1c level decreased after a 1-year follow-up owing to scheduled follow-up visits rather than additional metformin treatment [25].

For further evaluation, TIR, which generally describes the percent of time spent within the target glucose range $(3.9-10 \mathrm{mmol} / \mathrm{L})$, was also calculated from the CGM data. Yet, no notable difference was observed in TIR before and after the 3-month treatment between the two groups. Emerging as one of the central metrics of glycemic control, TIR has close association with vascular complications of diabetes [26, 27]. Although a goal of $>70 \%$ TIR was recommended by recent consensus statements for people with diabetes [28], baseline TIR of T1DM patients in the present study was considerably below target and was seemingly not improved by the adjuvant metformin therapy.

Tough robust data has suggested HbA1c and TIR are important predictors of the CVD risk in patients with diabetes [6, 26-29], but these metrics can only reflect the average glucose levels over a period of time. Further metrics are required to understand glycemic control in the entirety thereof, particularly those reflecting glucose fluctuations, such as hypoglycemia. Notably, hypoglycemia is a crucial barrier for patients with T1DM to achieve near-normal glycemic control [19,21]. Reports have indicated that T1DM patients with HbA1c levels of $<7.0 \%$ or $>7.5 \%$ suffered more frequent severe hypoglycemia than those with HbA1c levels of $7.0 \%$ to $7.5 \%$ [21]. GV, meanwhile, reflects the glucose homoeostasis over a given interval of time and has become prominent as another vital metric for assessing glycemic control in clinical practice. Further, GV supposedly contributes more to the onset of diabetic cardiovascular complications than persistent hyperglycemia. Prior research has demonstrated that acute glycemic fluctuations could lead to CVD through oxidative stress and nuclear factor- $x \mathrm{~B}$ activation [30,31]. Moreover, a positive association between intermittent high blood glucose exposure, endothelial dysfunction and damage has already been illustrated [32], while flattening GV has been reported to mitigate IR and reduce cIMT, a surrogate of CVD, in patients with T2DM [33]. As against T2DM, GV notably has more robust impact on diabetes complications in T1DM, most likely being attributed to the marked islet cell secretory dysfunction in the latter group[12].

As previously mentioned, the effect of metformin on HbA1c levels has been fully explored in patients with T1DM, while studies focusing on the aspect of GV are scarce. The other primary finding of the present study was that, in patients with T1DM, adjuvant metformin therapy reduced MAGE, together with other metrics of GV (SDBG, LAGE and CV). Similar reductions in MAGE and SD were also detected by Fei Gao et al. in patients with T2DM by employing metformin add-on insulin therapy[34]. Various metrics have been introduced to describe GV over the years, yet no consensus has been reached on the most appropriate characterization thereof. Introduced by Service et al.as the "gold standard" for the evaluation of the intraday GV, MAGE centers on major glycemic excursions rather than minor ones [35]. Dasari P S et al. reported that MAGE was closely linked with oxidative stress markers [36]. Further, a meta-analysis conducted by $\mathrm{Pu} \mathrm{Z}$ et al. revealed that a higher MAGE was associated with a higher risk of major adverse cardiovascular events (MACEs) in individuals, whether with or without diabetes [37]. However, no significant changes in MODD, which estimates between-day GV[38], were identified between the two groups in the present study. Since only 72 hours of CGM data were collected for quantification of GV in the present study, a longer CGM time might be required for the further evaluation of this metric.

Thus, the hypothesis in the present study is that metformin add-on insulin therapy could reduce glycemic fluctuation in T1DM to some extent, which may serve as one of the mechanisms of alleviating endothelium damage and enhance cardioprotective benefits. This hypothesis needs to be verified by prospective studies.

The final finding of the present study was that additional metformin decreased BMI and body weight by $0.2 \mathrm{~kg} / \mathrm{m} 2$ and $0.4 \mathrm{~kg}$ respectively. Bjornstad $\mathrm{P}$ et al. reported similar results in youth with T1DM [16], and even further reduction in BMI was detected by Agnieszka $Z$ et al. in adults with T1DM, in addition to excess body fat[17]. Moreover, adjuvant metformin therapy also reduced the insulin requirement in the present study. Lund et al. correspondingly identified a sustained reduction in weight and insulin dosage in T1DM patients over a one-year treatment with metformin [39]. All of the aforementioned research has indicated that additional metformin improved metabolic control while reducing IR in patients with T1DM. 
Be that as it may, in the present study, no improvement was observed in other cardiovascular risk factors, such as lipid profiles and blood pressure, in the MET+INS group. These results align well with previous research on youth with T1DM [16]. In contrast, conflicting conclusions were reported by Liu Y S et al., who found improvement in partial lipid profiles and diastolic blood pressure in T1DM patients with additional metformin[24].

According to the previous literature, metformin supposedly increases the risk of gastrointestinal adverse effects and may induce more hypoglycemia events in patients with T1DM [24, 40]. In the present study, no severe adverse events were observed and only 4 participants (11.8\%) in the metformin group had the insulin dose thereof down escalated due to gastrointestinal side effects. Further, all patients tolerated at least 1000mg metformin per day during the whole study, and metformin did not increase the incidence of hypoglycemia.

There are several significant strengths and limitations of the present study. First, this carefully designed, well-conducted clinical study is the first study to explore the effects of additional metformin on the GV in adults with T1DM. Second, both normal-weight and overweight/obese patients with T1DM were included, allowing further subgroup analysis in patients with different BMI levels. Third, the patients in both groups demonstrated relatively well adherence to CGM and had high visit attendance. The limitations in our study include the fact that the open-label design thereof may lead to a degree of bias. Moreover, CGM data were collected for 72 hours, while a recommendation of 2-4 weeks of data collection has been proposed by current guidelines in clinical practice $[28,41]$. This is because prolonging the duration of CGM could minimize the statistical bias of GV and TIR on the individual level. Additionally, the effect of individual diet and exercise deviations on GV could not be wholly excluded. The bias was minimized by reinforcing the guidance for diet and physical activity at each visit and urging the patients to maintain the same diet and physical activity during the CGM period.

\section{Conclusions}

Additional metformin therapy for 3 months reduced blood glucose fluctuation in adults with T1DM, particularly in those with higher BMI. Further, metformin improved the body composition and reduced the insulin requirement, indicating the reduction in insulin resistance in patients with T1DM. No severe adverse events or hypoglycemia were induced by additional metformin. To conclude, metformin as adjunctive therapy has potential prospects in the management of CVD risk in T1DM in the long term, which needs to be further elucidated in outcome trials.

Contribution statement

XZ conceived the study and wrote the paper. XZ and FY contributed to the design of the research. All authors were involved in data collection. DX, PX and SY analyzed the data. QZ and WY revised the manuscript. XZ and FY are the guarantors of this work. All authors edited and approved the final version of the manuscript.

References

1. Mayer-Davis, E.J., D. Dabelea, and J.M. Lawrence, Incidence Trends of Type 1 and Type 2 Diabetes among Youths, 2002-2012. N Engl J Med, 2017. 377(3): p. 301.

2. Rawshani, A., A. Rawshani, S. Franzen, et al., Mortality and Cardiovascular Disease in Type 1 and Type 2 Diabetes. N Engl J Med, 2017. 376(15): p. 1407-1418.

3. Livingstone, S.J., H.C. Looker, E.J. Hothersall, et al., Risk of cardiovascular disease and total mortality in adults with type 1 diabetes: Scottish registry linkage study. PLoS Med, 2012. 9(10): p. e1001321.

4. Livingstone, S.J., D. Levin, H.C. Looker, et al., Estimated life expectancy in a Scottish cohort with type 1 diabetes, 2008-2010.JAMA, 2015. 313(1): p. 37-44.

5. Cleary, P.A., T.J. Orchard, S. Genuth, et al., The effect of intensive glycemic treatment on coronary artery 
calcification in type 1 diabetic participants of the Diabetes Control and Complications Trial/Epidemiology of Diabetes Interventions and Complications (DCCT/EDIC) Study. Diabetes, 2006. 55(12): p. 3556-65.

6. Diabetes, C., G. Complications Trial Research, D.M. Nathan, et al., The effect of intensive treatment of diabetes on the development and progression of long-term complications in insulin-dependent diabetes mellitus. N Engl J Med, 1993. 329(14): p. 977-86.

7. Bloomgarden, Z. and G. Ning, Type 2 treatments for type 1 diabetes. J Diabetes, 2016. 8(5): p. 599-601.

8. Chillaron, J.J., J.A. Flores Le-Roux, D. Benaiges, et al., Type 1 diabetes, metabolic syndrome and cardiovascular risk. Metabolism, 2014. 63(2): p. 181-7.

9. Nadeau, K.J., J.G. Regensteiner, T.A. Bauer, et al., Insulin resistance in adolescents with type 1 diabetes and its relationship to cardiovascular function. J Clin Endocrinol Metab, 2010. 95(2): p. 513-21.

10. Schauer, I.E., J.K. Snell-Bergeon, B.C. Bergman, et al.,Insulin resistance, defective insulin-mediated fatty acid suppression, and coronary artery calcification in subjects with and without type 1 diabetes: The CACTI study. Diabetes, 2011. 60(1): p. 306-14.

11. Zhou, Z., B. Sun, S. Huang, et al., Glycemic variability: adverse clinical outcomes and how to improve it? Cardiovasc Diabetol, 2020. 19(1): p. 102.

12. Ceriello, A. and M.A. Ihnat, 'Glycaemic variability': a new therapeutic challenge in diabetes and the critical care setting. Diabet Med, 2010. 27(8): p. 862-7.

13. Klip, A. and L.A. Leiter, Cellular mechanism of action of metformin. Diabetes Care, 1990. 13(6): p. 696-704.

14. Cusi, K., A. Consoli, and R.A. DeFronzo, Metabolic effects of metformin on glucose and lactate metabolism in noninsulin-dependent diabetes mellitus. J Clin Endocrinol Metab, 1996. 81(11): p. 405967.

15. Effect of intensive blood-glucose control with metformin on complications in overweight patients with type 2 diabetes (UKPDS 34). UK Prospective Diabetes Study (UKPDS) Group. Lancet, 1998. 352(9131): p. 854-65.

16. Bjornstad, P., M. Schafer, U. Truong, et al., Metformin Improves Insulin Sensitivity and Vascular Health in Youth With Type 1 Diabetes Mellitus. Circulation, 2018. 138(25): p. 2895-2907.

17. Zawada, A., D. Naskret, P. Burchardt, et al., Metformin added to intensive insulin therapy improves metabolic control in patients with type 1 diabetes and excess body fat. Pol Arch Intern Med, 2018. 128(5): p. 294-300.

18. Petrie, J.R., N. Chaturvedi, I. Ford, et al., Cardiovascular and metabolic effects of metformin in patients with type 1 diabetes (REMOVAL): a double-blind, randomised, placebo-controlled trial. Lancet Diabetes Endocrinol, 2017. 5(8): p. 597-609.

19. Simmons, J.H., V. Chen, K.M. Miller, et al., Differences in the management of type 1 diabetes among adults under excellent control compared with those under poor control in the T1D Exchange Clinic Registry. Diabetes Care, 2013. 36(11): p. 3573-7.

20. Haller, M.J. and J.H. Silverstein, In pursuit of lower A1c. J Pediatr, 2009. 155(2): p. 161-2.

21. Weinstock, R.S., D. Xing, D.M. Maahs, et al., Severe hypoglycemia and diabetic ketoacidosis in adults with type 1 diabetes: results from the T1D Exchange clinic registry. J Clin Endocrinol Metab, 2013. 98(8): p. 3411-9.

22. Liu, C., D. Wu, X. Zheng, et al., Efficacy and safety of metformin for patients with type 1 diabetes mellitus: a meta-analysis.Diabetes Technol Ther, 2015. 17(2): p. 142-8. 
23. Libman, I.M., K.M. Miller, L.A. DiMeglio, et al., Effect of Metformin Added to Insulin on Glycemic Control Among Overweight/Obese Adolescents With Type 1 Diabetes: A Randomized Clinical Trial. JAMA, 2015. 314(21): p. 2241-50.

24. Liu, Y.S., C.N. Chen, Z.G. Chen, et al., Vascular and metabolic effects of metformin added to insulin therapy in patients with type 1 diabetes: A systematic review and meta-analysis. Diabetes Metab Res Rev, 2020: p. e3334.

25. Qiu, L., P. Ling, D. Yang, et al., Current status of metformin in addition to insulin therapy in adult patients with type 1 diabetes mellitus: An analysis from the Guangdong Type 1 Diabetes Mellitus Translational Medicine Study. J Diabetes, 2020.

26. Yoo, J.H., M.S. Choi, J. Ahn, et al., Association Between Continuous Glucose Monitoring-Derived Time in Range, Other Core Metrics, and Albuminuria in Type 2 Diabetes. Diabetes Technol Ther, 2020.

27. Beck, R.W., R.M. Bergenstal, T.D. Riddlesworth, et al., Validation of Time in Range as an Outcome Measure for Diabetes Clinical Trials. Diabetes Care, 2019. 42(3): p. 400-405.

28. Danne, T., R. Nimri, T. Battelino, et al., International Consensus on Use of Continuous Glucose Monitoring. Diabetes Care, 2017. 40(12): p. 1631-1640.

29. Intensive blood-glucose control with sulphonylureas or insulin compared with conventional treatment and risk of complications in patients with type 2 diabetes (UKPDS 33). UK Prospective Diabetes Study (UKPDS) Group. Lancet, 1998. 352(9131): p. 837-53.

30. Zhang, W., S. Zhao, Y. Li, et al., Acute blood glucose fluctuation induces myocardial apoptosis through oxidative stress and nuclear factor-kB activation. Cardiology, 2013. 124(1): p. 11-7.

31. Saisho, Y., Glycemic variability and oxidative stress: a link between diabetes and cardiovascular disease? Int J Mol Sci, 2014. 15(10): p. 18381-406.

32. Nusca, A., D. Tuccinardi, M. Albano, et al., Glycemic variability in the development of cardiovascular complications in diabetes. Diabetes Metab Res Rev, 2018. 34(8): p. e3047.

33. Liang, S., H. Yin, C. Wei, et al., Glucose variability for cardiovascular risk factors in type 2 diabetes: a meta-analysis. J Diabetes Metab Disord, 2017. 16: p. 45.

34. Gao, F., X. Ma, J. Peng, et al., The Effect of Acarbose on Glycemic Variability in Patients with Type 2 Diabetes Mellitus Using Premixed Insulin Compared to Metformin (AIM): An Open-Label Randomized Trial. Diabetes Technol Ther, 2020. 22(4): p. 256-264.

35. Service, F.J., G.D. Molnar, J.W. Rosevear, et al., Mean amplitude of glycemic excursions, a measure of diabetic instability.Diabetes, 1970. 19(9): p. 644-55.

36. Dasari, P.S., B.S. Gandomani, A.M. Teague, et al., Glycemic Variability Is Associated with Markers of Vascular Stress in Adolescents. J Pediatr, 2016. 172: p. 47-55 e2.

37. Pu, Z., L. Lai, X. Yang, et al., Acute glycemic variability on admission predicts the prognosis in hospitalized patients with coronary artery disease: a meta-analysis. Endocrine, 2020. 67(3): p. 526-534.

38. Molnar, G.D., W.F. Taylor, and M.M. Ho, Day-to-day variation of continuously monitored glycaemia: a further measure of diabetic instability. Diabetologia, 1972. 8(5): p. 342-8.

39. Lund, S.S., L. Tarnow, A.S. Astrup, et al., Effect of adjunct metformin treatment in patients with type-1 diabetes and persistent inadequate glycaemic control. A randomized study. PLoS One, 2008. 3(10): p. e3363.

40. Priya, G. and S. Kalra, A Review of Insulin Resistance in Type 1 Diabetes: Is There a Place for Adjunctive Metformin? Diabetes Ther, 2018. 9(1): p. 349-361. 
41. Battelino, T., T. Danne, R.M. Bergenstal, et al., Clinical Targets for Continuous Glucose Monitoring Data Interpretation: Recommendations From the International Consensus on Time in Range.Diabetes Care, 2019. 42(8): p. 1593-1603.

Table 1. Clinical characteristics of the study group $(n=65)$

\begin{tabular}{|c|c|}
\hline Variable & Value \\
\hline Sex, female/male, n (\%) & $36(55) / 29(45)$ \\
\hline Age (year) & $31 \pm 10$ \\
\hline Diabetes duration(year) & $8(2,15)$ \\
\hline Body weight $(\mathrm{kg})$ & $62.4 \pm 7.5$ \\
\hline $\operatorname{BMI}\left(\mathrm{kg} / \mathrm{m}^{2}\right)$ & $23.0 \pm 1.8$ \\
\hline Daily insulin dose ( $\mathrm{U} / \mathrm{kg}$ of body weight) & $0.63 \pm 0.09$ \\
\hline TG $(\mathrm{mmol} / \mathrm{L})$ & $1.98 \pm 0.62$ \\
\hline TC (mmol/L) & $5.19 \pm 0.78$ \\
\hline HDL-C (mmol/L) & $1.09 \pm 0.19$ \\
\hline LDL-C ( $\mathrm{mmol} / \mathrm{L})$ & $3.16 \pm 0.68$ \\
\hline SBP $(\mathrm{mm} \mathrm{Hg})$ & $117 \pm 10$ \\
\hline $\mathrm{DBP}(\mathrm{mm} \mathrm{Hg})$ & $74 \pm 7$ \\
\hline HbA1c (\%) & $8.1 \pm 0.9$ \\
\hline FBG $(\mathrm{mmol} / \mathrm{L})$ & $8.5 \pm 0.9$ \\
\hline 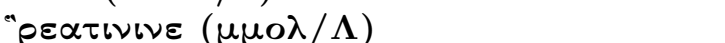 & $61.3 \pm 14.8$ \\
\hline $\operatorname{eGFR}\left(\mathrm{mL} / \mathrm{min} / 1.73 \mathrm{~m}^{2}\right)$ & $115.3 \pm 15.7$ \\
\hline
\end{tabular}

Normally distributed quantitative variables are presented as the mean \pm standard deviation, and nonnormally distributed quantitative variables are presented as the median (interquartile range, IQR). BMI, body mass index; TG, total triglyceride; TC, total cholesterol; HDL-C, high-density lipoprotein cholesterol; LDL-C, low-density lipoprotein cholesterol; SBP, systolic blood pressure; DBP, diastolic blood pressure; HbA1c, hemoglobin A1c; eGFR, estimated glomerular filtration rate.

Table 2. Baseline clinical characteristics of patients in two groups

\begin{tabular}{|c|c|c|c|}
\hline Variable & MET+INS & INS & $P$-Value \\
\hline Sex, female/male, n (\%) & $18(56) / 14(44)$ & $18(60) / 12(40)$ & 0.767 \\
\hline Age (year) & $31 \pm 10$ & $32 \pm 11$ & 0.583 \\
\hline Diabetes duration (year) & $8(2,12)$ & $9(3,20)$ & 0.237 \\
\hline Body weight $(\mathrm{kg})$ & $62.6 \pm 7.6$ & $62.2 \pm 7.6$ & 0.814 \\
\hline Daily insulin dose ( $U / \mathrm{kg}$ of body weight) & $0.62 \pm 0.07$ & $0.64 \pm 0.10$ & 0.315 \\
\hline BMI $\left(\mathrm{kg} / \mathrm{m}^{2}\right)$ & $23.0 \pm 1.8$ & $22.9 \pm 1.8$ & 0.917 \\
\hline TG $(\mathrm{mmol} / \mathrm{L})$ & $2.11 \pm 0.61$ & $1.83 \pm 0.60$ & 0.066 \\
\hline TC $(\mathrm{mmol} / \mathrm{L})$ & $5.24 \pm 0.64$ & $5.13 \pm 0.91$ & 0.574 \\
\hline HDL-C (mmol/L) & $1.12 \pm 0.19$ & $1.05 \pm 0.18$ & 0.159 \\
\hline LDL-C (mmol/L) & $3.20 \pm 0.55$ & $3.12 \pm 0.80$ & 0.652 \\
\hline $\mathrm{SBP}(\mathrm{mm} \mathrm{Hg})$ & $117 \pm 10$ & $117 \pm 9$ & 0.789 \\
\hline DBP (mm Hg) & $72 \pm 7$ & $75 \pm 8$ & 0.210 \\
\hline HbA1c (\%) & $8.2 \pm 0.8$ & $8.0 \pm 0.9$ & 0.246 \\
\hline 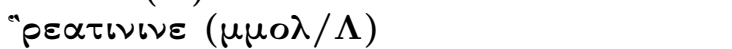 & $64.0 \pm 16.6$ & $58.4 \pm 12.4$ & 0.137 \\
\hline eGFR $\left(\mathrm{mL} / \mathrm{min} / 1.73 \mathrm{~m}^{2}\right)$ & $112.6 \pm 18.4$ & $118.1 \pm 11.9$ & 0.172 \\
\hline MAGE (mmol/L) & $6.88 \pm 2.75$ & $5.48 \pm 3.27$ & 0.073 \\
\hline LAGE ( $\mathrm{mmol} / \mathrm{L})$ & $11.53 \pm 3.63$ & $10.13 \pm 5.09$ & 0.216 \\
\hline SDBG $(\mathrm{mmol} / \mathrm{L})$ & $2.85 \pm 0.98$ & $2.47 \pm 1.26$ & 0.184 \\
\hline $\operatorname{MODD}(\mathrm{mmol} / \mathrm{L})$ & $3.11 \pm 1.31$ & $3.13 \pm 1.61$ & 0.957 \\
\hline
\end{tabular}




\begin{tabular}{llll} 
CV (\%) & $27.94 \pm 10.88$ & $24.44 \pm 11.23$ & 0.218 \\
TIR (\%) & $45.80 \pm 21.19$ & $53.67 \pm 24.37$ & 0.179 \\
MSG (mmol/L) & $10.50 \pm 1.47$ & $9.97 \pm 1.56$ & 0.175 \\
\hline
\end{tabular}

Normally distributed quantitative variables are presented as the mean \pm standard deviation, and nonnormally distributed quantitative variables are presented as the median (interquartile range, IQR). BMI, body mass index; TG, total triglyceride; TC, total cholesterol; HDL-C, high-density lipoprotein cholesterol; LDL-C, low-density lipoprotein cholesterol; SBP, systolic blood pressure; DBP, diastolic blood pressure; HbA1c, hemoglobin A1c; eGFR, estimated glomerular filtration rate; MAGE, mean amplitude of glucose excursions; LAGE, largest amplitude of glycemic excursions; SDBG, standard deviation of blood glucose ; MODD, mean of daily differences; CV, coefficient of variation; TIR, time in range; MSG, mean sensor glucose.

Table 3. The effects of MET+INS on changes in glycemic variability and glycemic control compared with INS after 3-month intervention

\begin{tabular}{llll}
\hline & MET + INS $(\mathrm{n}=32)$ & INS $(\mathrm{n}=30)$ & $P$-Value \\
\hline Glycemic variability & Glycemic variability & Glycemic variability & Glycemic variability \\
${ }^{M} A G E(\mathrm{mmol} / \mathrm{L})$ & $-1.47 \pm 3.39$ & $1.05 \pm 4.24$ & 0.012 \\
${ }^{S} D B G(\mathrm{mmol} / \mathrm{L})$ & $-0.62 \pm 1.15$ & $0.08 \pm 1.23$ & 0.023 \\
${ }^{S} \mathrm{AGE}(\mathrm{mmol} / \mathrm{L})$ & $-2.28 \pm 4.71$ & $1.77 \pm 5.71$ & 0.003 \\
${ }^{C} V(\%)$ & $-6.08 \pm 12.31$ & $2.29 \pm 11.57$ & 0.008 \\
${ }^{M} O D D(\mathrm{mmol} / \mathrm{L})$ & $-0.27 \pm 1.93$ & $0.14 \pm 2.43$ & 0.461 \\
Glycemic control & Glycemic control & Glycemic control & Glycemic control \\
${ }^{T} I R(\%)$ & $-3.57 \pm 13.49$ & $-3.30 \pm 17.16$ & 0.944 \\
${ }^{M} S G(\mathrm{mmol} / \mathrm{L})$ & $-0.13 \pm 0.58$ & $0.23 \pm 0.75$ & 0.038 \\
${ }^{H} B A 1 \mathrm{c}(\%)$ & $-0.05 \pm 0.22$ & $0.03 \pm 0.28$ & 0.217 \\
\hline
\end{tabular}

Delta $\left({ }^{\prime}\right.$ value $=3-$ monthvalue - -baselinevalue.

Variables are expressed as the mean \pm standard deviation. MAGE, mean amplitude of glucose excursions; SDBG, standard deviation of blood glucose; LAGE, largest amplitude of glycemic excursions; CV, coefficient of variation; MODD, mean of daily differences; TIR, time in range; MSG, mean sensor glucose; HBA1c, hemoglobin A1c.

Table 4. Subgroup analysis of glycemic variability and glycemic control in the MET+INS group

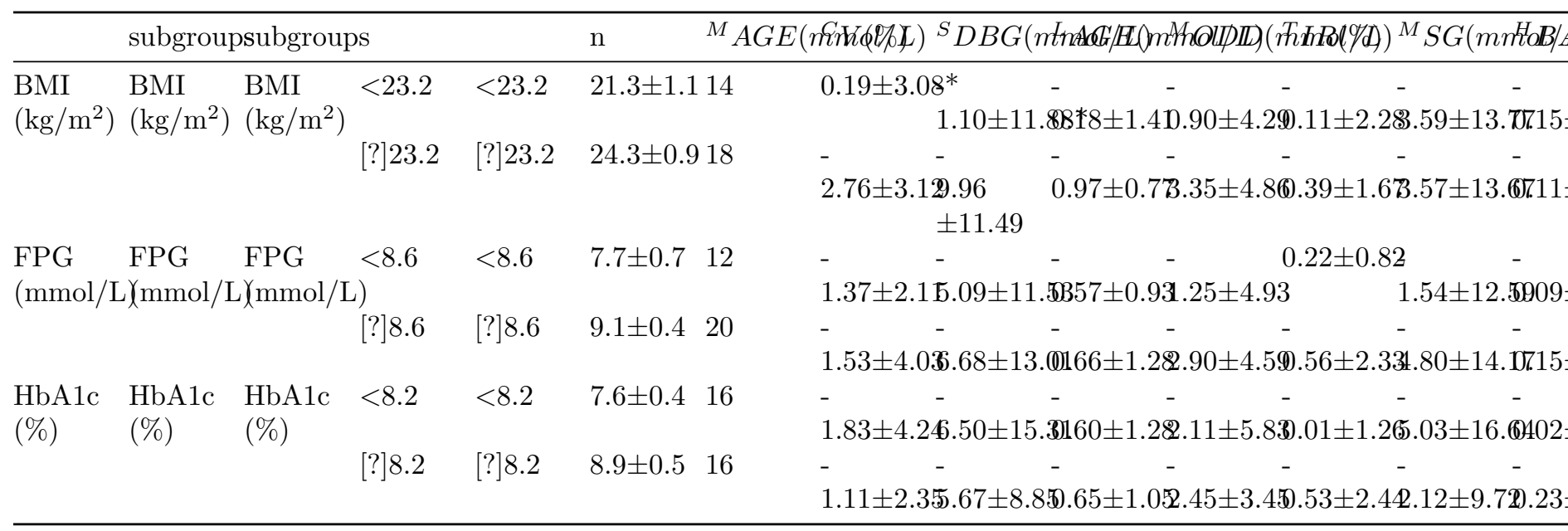


Data are mean \pm standard deviation; BMI, body mass index; FPG, fasting plasma glucose; HbA1c, glycated hemoglobin A1c;

*, $P<0.05$ compared within subgroups.

Table 5. The effects of MET+INS on changes in lipid profiles and other parameters compared with the INS after 3-month intervention

\begin{tabular}{|c|c|c|c|}
\hline & $\mathrm{MET}+\mathrm{INS}(\mathrm{n}=32)$ & INS $(n=30)$ & $P$-Value \\
\hline${ }^{T} G(m m o l / L)$ & $-0.23 \pm 0.62$ & $-0.13 \pm 0.55$ & 0.520 \\
\hline${ }^{T} C(\mathrm{mmol} / L)$ & $0.03 \pm 0.15$ & $0.04 \pm 0.24$ & 0.899 \\
\hline${ }^{H} D L-$ & $0.11 \pm 0.22$ & $0.07 \pm 0.21$ & 0.473 \\
\hline \multicolumn{4}{|l|}{$C(\mathrm{mmol} / L)$} \\
\hline \multicolumn{4}{|l|}{$C(\mathrm{mmol} / L)$} \\
\hline$B$ odyweight $(\mathrm{kg})$ & $-0.4 \pm 0.6$ & $0.2 \pm 0.5$ & ¡0.001 \\
\hline${ }^{B} M I$ & $-0.2 \pm 0.2$ & $0.1 \pm 0.2$ & ¡0.001 \\
\hline \multicolumn{2}{|c|}{${ }^{D}$ ailyinsulindose $(U / k g @ f(0)+4$ wolight $)$} & $0.00 \pm 0.02$ & ¡0.001 \\
\hline${ }^{S} B P$ & $-1 \pm 9$ & $0 \pm 9$ & 0.665 \\
\hline${ }^{D} B P$ & $1 \pm 7$ & $-1 \pm 8$ & 0.369 \\
\hline
\end{tabular}

TG, total triglyceride; TC, total cholesterol; HDL-C, high-density lipoprotein cholesterol; LDL-C, low-density lipoprotein cholesterol; BMI, body mass index; SBP, systolic blood pressure; DBP, diastolic blood pressure.

Figure Legends

Figure 1. The diagram of clinical trial in participants with type 1 diabetes mellitus

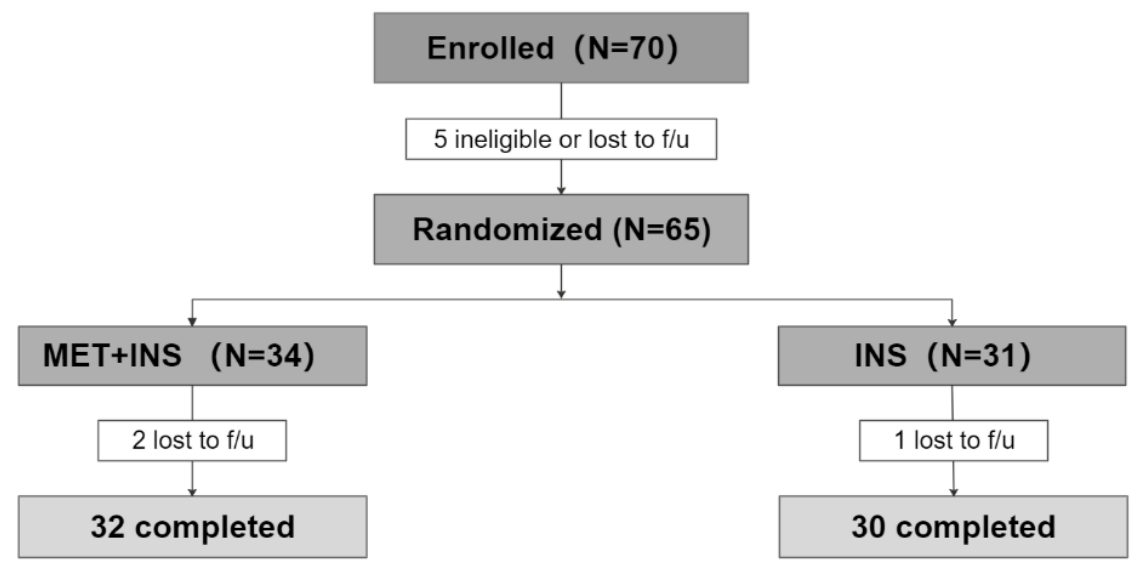

f/u, follow-up.

Figure 2. Metformin reduced glycemic variability in patients with type 1 diabetes mellitus. 
A

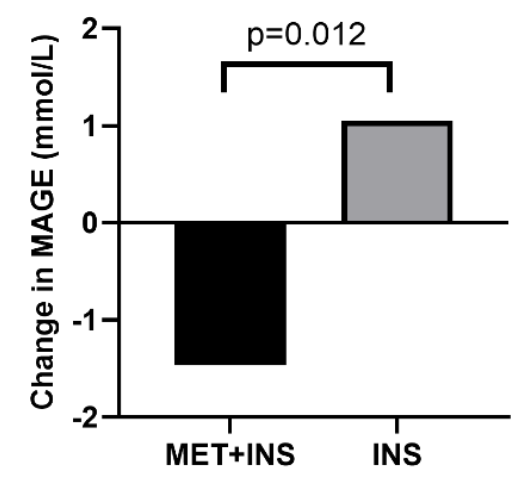

C

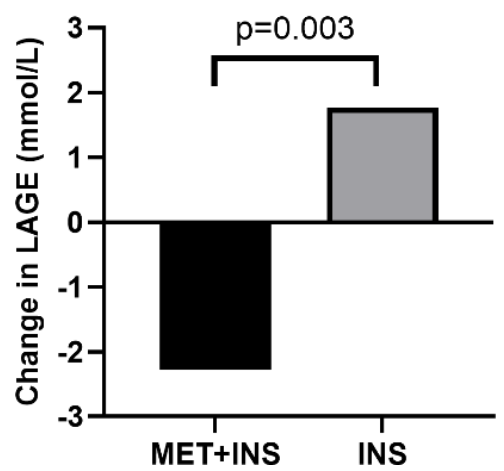

B
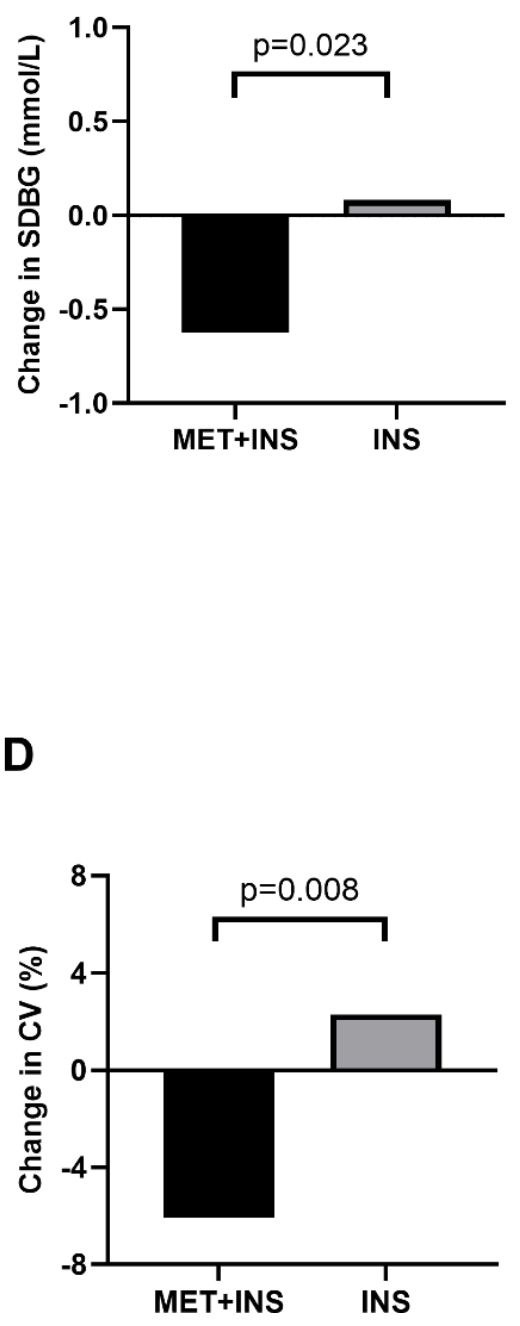

A, Change in mean amplitude of glucose excursions (MAGE; mmol/L) in response to MET+INS vs INS.

$\mathrm{B}$, Change in standard deviation of blood glucose (SDBG; mmol/L) in response to MET+INS vs INS.

C, Change in largest amplitude of glycemic excursions (LAGE; mmol/L) in response to MET+INS vs INS.

$\mathrm{D}$, Change in coefficient of variation $(\mathrm{CV} ; \%)$ in response to MET+INS vs INS.

Compared with the INS, MET+INS improved the metrics of glycemic variability (MAGE, SDBG, LAGE, CV) over 3-month intervention.

Figure 3. Metformin reduced insulin requirement andimproved body composition in patients with type 1 diabetes mellitus. 
A

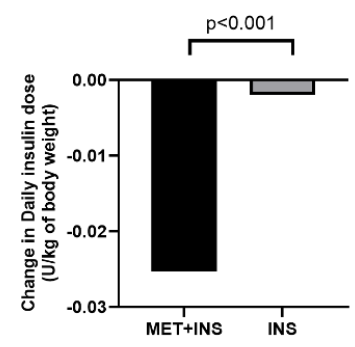

B

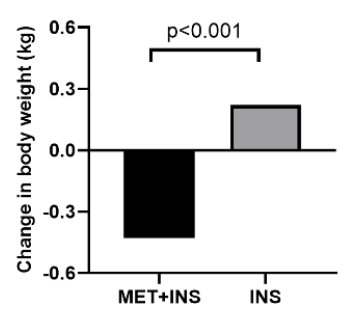

C

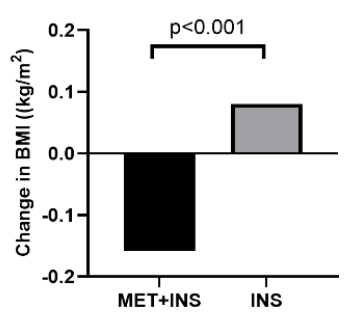

A, Change in daily insulin dose ( $\mathrm{U}$ per kg of body weight) in response to MET+INS vs INS. B, Change in body weight (kilograms) in response to MET+INS vs INS. C, Change in body mass index (BMI; kilograms per meter squared) in response to MET+INS vs INS.

Significant reductions in daily insulin dose, body weight as well as BMI were observed in the patients with MET+INS, and the changes were significantly different in comparison with those with INS.

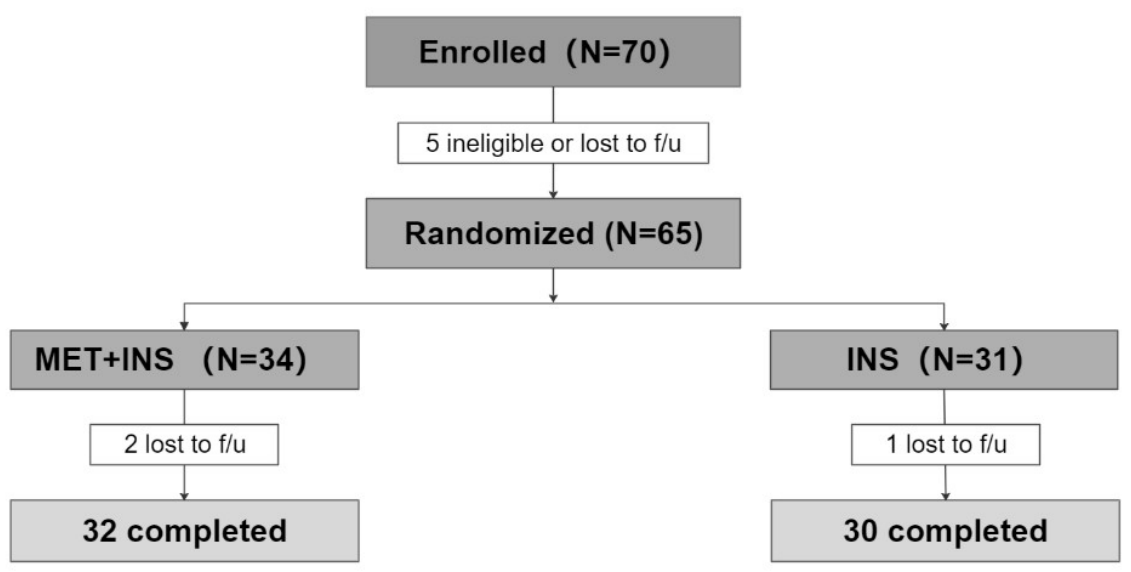


A

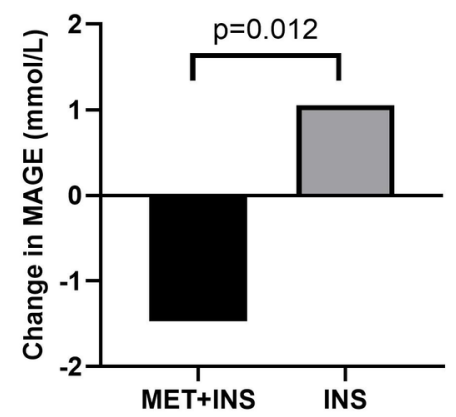

C

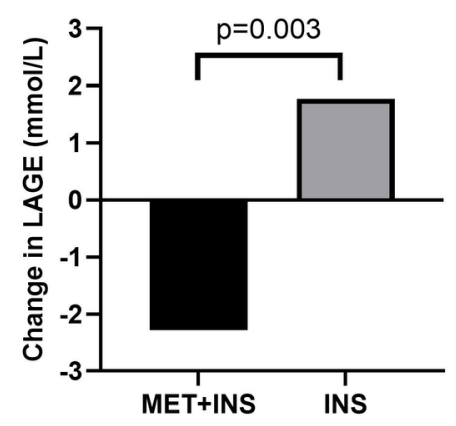

B

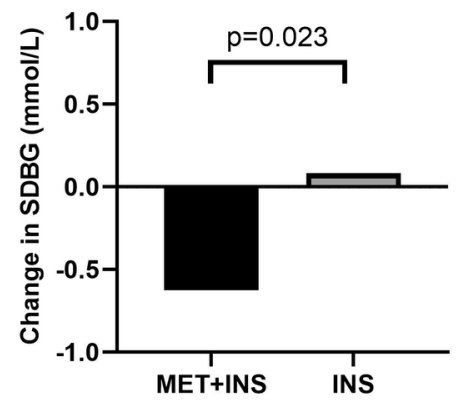

D

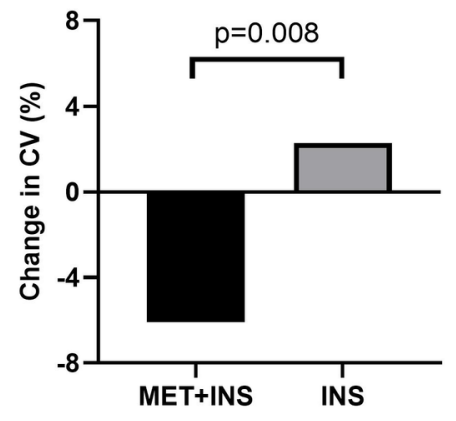

A

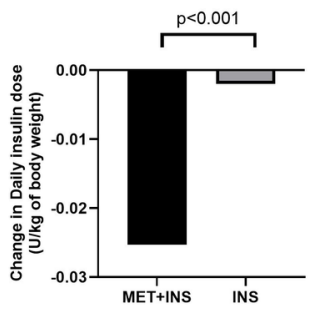

B

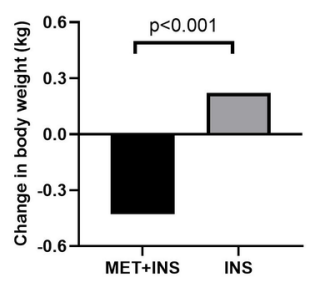

C

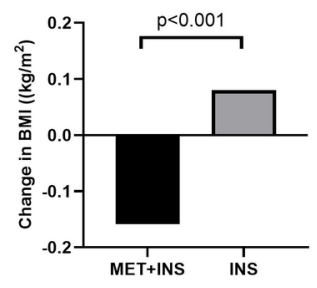

